mètres. Elles sont prévues pour n’avoir pas à travailler à plus de $4^{2} \mathrm{kgs}$ par $\mathrm{cm}^{2}$. Le rapport de leur hauteur à leur diamètre est égal à 13,5 .

Les moules des colonnes sont constitués par des tôles de $3,2 \mathrm{~mm}$. d'épaisseur, raidies par des cornières de $50,8 \mathrm{~mm}$. de côté. Les moules des parois circulaires sont en bois, bien dressés.

Les moules du toit étaient suspendus à des poutrelles de fer, du type ordinairement employés dans la construction des bâtiments, de manière à pouvoir être utilisés ultérieurement après achèvement des travaux sans trop de perte. Ces poutrelles de fer reposaient sur les colonnes complètement terminées. Comme les barres métalliques de renforcement des poutres du toit sont noyés dans les chapiteaux des colonnes, de manières que ces quatre poutres qui aboutissent à une même colonne soient solidaires les unes des autres, il se trouve que les poutres sont en somme en deux pièces. Le béton constituant l'encastrement dans les chapiteaux des colonnes étant coulé et ayant fait prise avant la confection du corps de la poutre proprement dit (1).

Les moules du toit étaient en nombre suffisant pour servir “ la couverture d'un quart du réservoir. Au fur et à mesure de l'avancement, ils étaient déplacés, de sorte que chaque moule a servi 16 fois de suite pour l'ensemble des 4 réservoirs.

Pour faciliter le déversement du béton sur les divers points du chantier, on fit emploi d'un pont tournant métallique, qui s'appuyait d'un côté sur la tour centrale, autour de laquelle il pivotait, et de l'autre côté sur une voie de roulement circulaire, spécialement aménagée à l'extérieur de la paroi verticale. Le même pont a servi successivement pour les quatre réservoirs.

Le béton employé était dosé à raison de I de ciment pour 3 de sablc et 3 de graviers ou de pierres cassées. Le mètre cube de béton, y compris le renforcement métallique, et les moules, est revenu à 50 francs environ.

Le premier réservoir a été mis en service au début de l'année 1909 .

\section{L'INDUSTRIE DE L'ACIDE NITRIQUE EN NORVEGE}

Communicatıon de M. $S_{1}$ Eyde à l'assnciatıon des ingénieurs et architectes norvéglens (*)

J'avais affirmé il y trois ans, à cette même place, devant l'Association polytechnique, que la découverte de M. Birkeland et de moi offrait une base à une nouvelle industrie. Je puis dire maintenant que celle-ci a fourni aujourd'hui, au point de vue économique, la preuve de sa viabilité. Jusqu'à présent, on a appliqué à l'industrie norvégienne de l'acide

(I) Des essais préalables avaient été effectués pour se rendre compte de la diminution de résistance que cette double confection pouvait entrainer. Deux poutres coulées d'un seul bloc, tt deux poutres coulées en deux fois turent essayées par Hexion, en reposant sur deux appuis disposés près de leurs extrémités, où se trouvaient la jonction des deux coulées, D'après ces essais, la confection en deux fors n'apporterait aucune diminution de résistance (c'est le contraire qui a été constaté!), toutefois, les conditions des essais ne sont pas tout à fait les mêmes que celles des réservoirs, où les poutres sont soumises, aux points de soudure, à un moment fléchissant d'encastrement qui n'existait pas dans les essais.

(*) Aftenposten: 13 février 1909. - Zeitschrift für Elecktrochimie, 1er mars 1909 nitrique, pour les établissements de Nottoden Svalgfos, Rjukan et Vamma, la somme de 22 millions de couronnes, sur lesquels 17 millions et demi ont été apportés par les ouvriers, les usiniers et les commerçants norvégiens. A la fin de 1910, ces établissements seront achevés. Leur prix total at teindra 54 millions de couronnes, dont 42 , millions seront dus à la Norvége.

La question de la concurrence des produits de cette indus. trie avec le salpètre du Chili ne se pose pas, car la consommation de l'azote croit si rapidement que la quantité que nous pouvons produire en Norvège n'aura de long. temps aucune influence sur le marché.

Nous sommes en voie d'aménager dans notre pays, pour la production des oxycies de l'azote, des forces hydraulicques d'une puissance de 500.000 chevaux, qui pourront livrer annuellement 300.000 tonnes dacide nitrique. Les deux fabriques nécessaires pour cela, chacune de la force de celle de Rjukan actuellementen construction, ne seront pas en activité avant moins de 10 ans; elles exigeront l'emploi d'un capital de 150 millions de couronnes au total.

Le Chili exporte acluellement 1,8 million de tonnes de salpètre par an. Dans l'année 1920, l'exportation en sera vraisemblablement de 2,5 millions de tornes. A la mîme époque, l'exportation annuelle de la Norvège atteindra 300.000 tonnes, valant 45 millions de couronnes, ce qui correspond à 12 pour 100 de l'exportation du Chili. La crainte que, par réduction du prix du salpètre du Chili, les conditions économiques d'exploitation du procédé Norvégien ne soient troublés, est vaine; car, au Chili, le climat et les cilconstances opposent au travail de production du salpètre de soude toutes les difficultés imaginables, ce qui exclue tout avilissement sensible du prix de vente au dessous des prix actuels. Lorsque l'établissement en construction à Rjukan sera terminé, une nouvelle ville ouvrière s'élèvera près de Saaheim pour 1.000 hommes, c'est à-dire pour environ 4.000 habitants. Les sociétes intéressées à l'affaire et les autorités prendront soin que les ouvriers et leur's familles y puissent vivre dans les conditions les plus favorables.

En ce qui concerne Nottoden, il s'est présenté au commencement de la fabrication des difficultés qu'on n'avait guère pu prévoir. Trois mois après la mise en marche, les générateurs furent brûlés. Un comité des premiers électriciens d'Europe fut convoqué, qui arrivèrent à celte conclusion que des fautes de construction avaient été commises, dont la correction élait cependant possible. De pareilles surprises n'ont pas manqué du reste en d'autres endroits. Les générateurs furent modifiés. Depuis la fin des réparations, tout est demeuré en ordre, et les machines tournent depuis neuf mois sans interruption. Naturellement, l'incident des générateurs et quelques autres dans les conducteurs et les tableaux ont diminué la production du début. Malgré tout, nous avons réussi à obtenir pour notre entreprise à Nottoden un résultat financier favorable.

Notre exploitation a clonné un revenu net d'un demi-million de couronnes pour une recette totale de 2 millions, les dépenses pour exploitation, amortissement et frais généraux atteignent 1 million et demi. Les arrêts déjà signalés à la fabrique et à l'usine génératrice ne permirent pas, en 1908, une marche moyenne de plus de $13.000 \mathrm{kw}$., alors que l'installation est construite pour une puissance normale de $22.000 \mathrm{kw}$.Nous fúmes, pendant les six derniers mois, à part quelques périodes de basses eaux, en pleine marche régulière, et avons alor's en moyenne travaillé avec $19.500 \mathrm{kw}$. soitavec $2.500 \mathrm{kw}$. de moins qu'en marche théorique. Pendant cette durée, nous avons atteint une recette de 1.500 .000 couronnes pour une dépense de 970.000 couronnes, c'est.àdire un revenu net, pour 6 moli; de 530.000 couronnes, qui représente, en calculant pour un an, 1.060.c00 couronnes. Ces recettes rémunèrent un capital de 11 millions de coutronnes pour les deux usines de Svalgfoss et Nottoden. Avec 
la mise en fabrication du nitrate d'ammoniaque et du nitrate de polasse, qui sont à l'étude, les recettes s'élèveront, ainsi que par la régularisation projetée des forces hydrauliques et par la mise en service des nouvelles installations de Mjosvand et de Maarvand.

Je ferai remarquer,particulièrement, que ces résultats ont élć obtenus avec une industrie entièrement nouvelle, qui fa pu sappuyer sur aucun exemple, et que l'exercice écoulé est le premier exercice d'exploitation de la nouvelle industrie. Si lon compare ces résultats avec les calculs laifs autrefois, il en ressort que nos rendements sont un peu meilleurs, et nos dépenses un peu plus faibles que nous ne l'avions admis.

Permettez-moi quelques remarques au sujet de la genèse de cette affaire. J'assumai moí-même les premiers frais des peherches avec quelques amis, parmi lesquels la plupart se retirèrent peu à peu, car ils crurent l'entreprise trop andacieuse. Pour la continuation des recherches, on fonda ensuite la société Det Norske Kralstofkompagnie, au capital de 500.000 couronnes; il ressotit bientôt qu'elle ne suffisait pas pour l'exécution de recherches coùteuses et de grande tchelle. En même temps, on reconnut que l'exécution de ces recherches serait considérablement facilitée, au cas ou nous disposerions, non-seulement de notre invention, mais encore de chûtes d'eau qui représenteraient une certaine valeur, mème après un échec de la méthode, et qui, en cas do succès,étaient susceptibles de croître extraordinairement de valeur en accroissant celle du procédé. Pour exécuter cette combinaison de possession de brevets et de chutes d'eau, la Société Det. Norske A ktieselskab for elektrokemisk Industrie, au capital de 5 millions de couronnes, fut fondée, avec le soutien précieux de $M$. Wallenberg (célébre banquier suédois) dont le mérite, tant par le risque qu'il courut que pour le travail personnel énorme qu'il fournit pour ledéveloppement de notre eutreprise norvégienne, ne peut être prisé trop haul. Cette nouvello Société acheta la majorité des actions dos sociétés existantes : Rjukan foss, Wamma. fossekompagnie, et Arendalsfossekompagnie (Boilefoss), ainsi que de la Det Norshe Kvalstofkompagnic, qui possédait les brevets de Birkeland et Eyde et quelçues autres, concernant l'utilisation de l'azote atmosphérique. lille me lournit les moyens de construire à Notodden une petite falsrique qui fut prête en 1904 , et ello me chargea d'étudicr la méthode. Les dépenses totales pour les recherches ont dépassé le chiffre de 1.000 .000 couronnes.

Lorsque les résultats obtenus dans cette première usine d'essais nous eurent pleiuement convaincu que le procédé était utilisable industriellement, nous commençàmes à penser à lutilisation du dit procédé sur une plus grande échelle, et, dans le but de construire une nouvelle fabrique plus grande à Notodden, et d'utiliser les forces dont nous disposions à Svalgfoss, fut alors fondée la Norsk hydroelektrisk Kualssotaktieselskab, au capital-actions de 7.500000 couronnes. Celle grosse fabrique fut prète à lautomne $190 i$, tandis que la petite fabrique donnait toujours de meilleur's risultats. A ce moment, nous vimes que l'oxé. culion de notre plan daus loute són extension exigeait des capitaux qu'il était difficile de réussir à trouver en Norvège, linus ne pouvions pas compter nous procurer la somme necessaire pour l'installation de 250000 HP i Rjukan dans intre pays seul.

Précisément, en ces conjonctures, nous apprìmes la réusste de la Badische Amilin und Soda Fabrik dans la même vole. Nous nous réunimes avec cetteentreprise, et il fut facile aux Sociétés réunies de s'assurer la somme totale qui était nécossaire à l'acquisition et à l'aménagement de la cliute de lijulian, et éventuellement dautres forces hydrauliques. lour des raisons d'ordre pratique, il fut utile de former deux nouvelles Sociétes, dont l'une se proposait l'installalion des forces et livrait du courant, tandis que l'autre ab. sorbait ce courant, et se chargeait de la fabrication. Ces deux nouvelles Sociétés reçurent les noms de Norsk kraftaklieselskab, au capital de 16 millions de couronnes, et Aktieselskabet det Norike Salpelerveskel o, au capital de 18 millions de couronnes. Par suite d'entente entre nos entreprises allemande et norvégicnne, les résultats d'essais dune nouvelle, fabrique en construction près de Nolodden, décideront si, pour la grande installation finale, ce sont les fours de la Badische Anilin und Soda Fabrich ou ceux de Birkeland et Eyde qui seront employés.

L'utilisation des chutes de Rjukan exige la création d'une ligne de transport de la ville de Skien jusqu à Rjukan. Elle se compose d'une ligne de bateau de Skien à Notodden. d'une voie ferrée jusqu'à Tinneset, d'un trajet jusqu'ì. Vestljorddalen. et d'une voie ferrée pal celte vallée jusqu'a Rjulian. Les conditions de concessinn de la ville intèresséc, pour le chemin de fer, le droit de cession de l'Etat. et d'autres circonstances encore, obligent à la fondation dune troisième société indépendante au capital de 3 millions de couronnes, qui s'occupera de l'établissement de ces diverses lignes de transport sous le nom de Norsk Transporlaktieselskab.

\section{RËGLEMENTATION DES COMPTEURS}

Le ministre destravaux publics, des postes et des télégraphes, Vu la loi du 15 juin 1906 sur les distributions d'énergie (*);

$\mathrm{Vu}$ les articles 6 des cahiers des charges types des distribulions publiques d'énergie électrique en date des $17 \mathrm{mai}$ et 20 août $\left.\lg 9 \mathbf{8}^{(\star *}\right)$;

Vu l'avís du comité d'électricite,

Arrête:

Les compteurs servant à mesurer les quantités d'énergie électriques livrées au public par les concessionnaires ou permissionnaires de distributions publiques d'énergie électrique soumises aux clauses et ronditions des cahiers des charges types en date des 17 mai et 20 aout 1908 , devront satisfaire, par application de l'article i 6 desdits cahiers des charges, aux conditions ci-après énumérées:

Article premier. - Définition du trpe. - Le type de compteur est défini par ses dessins de construction.

Sont considérés comme de même type les compteurs de calibres différents, construits sur les mêmes dessins et dont les différences ne portent que sur les bobinages jui restent, d'ailleurs, semblablement placés.

Le type peut comporter l'emploi d'appareils accessoires, tels que transformateurs, etc., ces accessoires forment partie integrante du compteur.

Chaque type de compteur porte un nom; si le même nom s'applique a plusieurs calibres du même type, chaque rype porte, en outre, un numéro de série caractéristique. Le nom et le numéro de série figurent sur les plaques des appareils mis en service.

Aвт. 2. - Constitution du dossier de demandc d'approbation. - Le dossier de demande d'approbation contient les pièces suivantes:

I" Les dessins dexécution à des échelles suffisantes pour en permettre la lecture facile;

$2^{\circ}$ Une note descriptive exposant le principe du compteur, décrivant son mécanisme et son fonctionnement, indiquant la manière dont il est parć, dans la mesure du possible, aux différentes causes d'erreur. Cette note doit, en outre:

a) Indiquer le détail des bobinages que peut recevoir le type, et les calibres correspondants;

() Voir La Houlle Blanche de jullet 1006.

(") Voir La Foulle Blanche de septembre et novembre 1908. 